\title{
Critical dimensions and higher order Sobolev inequalities with remainder terms*
}

\author{
Filippo Gazzola \\ Dipartimento di Scienze T.A. - via Cavour 84, 15100 Alessandria, Italy \\ Hans-Christoph Grunau \\ Fachgruppe Mathematik, Universität, 95440 Bayreuth, Germany
}

\begin{abstract}
Pucci and Serrin [21] conjecture that certain space dimensions behave "critically" in a semilinear polyharmonic eigenvalue problem. Up to now only a considerably weakened version of this conjecture could be shown. We prove that exactly in these dimensions an embedding inequality for higher order Sobolev spaces on bounded domains with an optimal embedding constant may be improved by adding a "linear" remainder term, thereby giving further evidence to the conjecture of Pucci and Serrin from a functional analytic point of view. Thanks to Brezis-Lieb [5] this result is already known for the space $H_{0}^{1}$ in dimension $n=3$; we extend it to the spaces $H_{0}^{K}(K>1)$ in the "presumably" critical dimensions. Crucial tools are positivity results and a decomposition method with respect to dual cones.
\end{abstract}

\section{Sharp higher order Sobolev inequalities and critical dimensions}

In a celebrated paper, Pucci-Serrin [21] studied the following critical growth problem for polyharmonic operators

$$
\left\{\begin{array}{l}
(-\Delta)^{K} u=\lambda u+|u|^{K_{*}-2} u \quad \text { in } \Omega \\
D^{k} u=0 \quad \text { on } \partial \Omega \quad k=0, \ldots, K-1
\end{array}\right.
$$

${ }^{*}$ This work was supported by the Vigoni-programme of CRUI and DAAD 
where $K \in \mathbb{N}, \Omega \subset \mathbb{R}^{n}(n \geq 2 K+1)$ is an open bounded domain with smooth boundary and $K_{*}=\frac{2 n}{n-2 K}$ is the critical Sobolev exponent (the largest $q$ for which the embedding $H_{0}^{K}(\Omega) \subset L^{q}(\Omega)$ is continuous); with this notation $1_{*}=2^{*}=\frac{2 n}{n-2}$. Further, $D^{k} u$ denotes any derivative of $u$ of order $k$. Pucci-Serrin were interested in existence of nontrivial radial solutions of (1) when $\Omega=B_{1}$, the unit ball in $\mathbb{R}^{n}$; their interest was motivated by previous results from the famous paper by Brezis-Nirenberg [6] who studied the corresponding problem in the case $K=1$ and showed a striking difference between the space dimension $n=3$ and the higher dimensions $n \geq 4$. More precisely, if $\lambda_{1}$ denotes the first eigenvalue of $-\Delta$ in $B_{1}$, it is shown in [6] that (1) admits a positive radial solution if and only if $\lambda \in\left(0, \lambda_{1}\right)$ when $n \geq 4$, while it admits a positive radial solution if and only if $\lambda \in\left(\lambda_{1} / 4, \lambda_{1}\right)$ when $n=3$. Thus, one interesting problem concerning (1) is to determine the constant $\lambda^{*} \geq 0$ for which the relation $\lambda>\lambda^{*}$ is a necessary condition for the existence of a nontrivial radial solution of (1) in $B_{1}$. Pucci-Serrin [21] call critical dimensions the space dimensions for which $\lambda^{*}>0$ and conjecture, for a given $K \geq 1$, that the critical dimensions are $n=2 K+1, \ldots, 4 K-1$ : by the results in [6] this conjecture was already proved for $K=1$, next it has been proved for $K=2,3,4$ (see $[3,8,13,21,22]$ ); moreover, it is known that for all $K \geq 1$ the dimensions $n \geq 4 K$ are not critical, see [14]. Some time later, Grunau [15] defined weakly critical dimensions as the space dimensions for which a necessary condition for the existence of a positive radial solution of (1) in $B_{1}$ is $\lambda \in\left(\lambda^{*}, \lambda_{1}\right)$ for some $\lambda^{*}>0$ : he showed that weakly critical dimensions are precisely $n=2 K+1, \ldots, 4 K-1$. So, at least a weakened version of the PucciSerrin conjecture could be shown, a full proof of their original conjecture will be considerably more difficult, if possible at all. It remains to understand better the role of these dimensions.

The semilinear eigenvalue problem (1) also has an intrinsic interest in more general domains. By means of a generalized Pohožaev identity, Pucci-Serrin [20] proved that (1) only admits the trivial solution $u \equiv 0$ whenever $\lambda<0$ and $\Omega$ is starshaped. Moreover, for any bounded smooth domain $\Omega$, it is shown in $[8,12,14,22]$ that nontrivial solutions exist under different assumptions in the cases $n \geq 4 K$ and $n<4 K$. This shows that critical dimensions may not only be a phenomenon of radial symmetry in balls (cf. also [6, Theorem 1.2"]); some attempts [16, 18] 
were made in order to explain this phenomenon by means of the local summability properties of the fundamental solution of the operators involved.

Motivated by the non-existence results in [6], Brezis-Lieb [5] proved the following sharp Sobolev inequality in bounded open sets for the space $H_{0}^{1}$ :

$$
\|f\|_{1}^{2} \geq S|f|_{1_{*}}^{2}+C|f|_{\frac{n}{n-2}, w}^{2} \quad \forall f \in H_{0}^{1}(\Omega) .
$$

Here $\|f\|_{1}^{2}:=\int_{\Omega}|\nabla f|^{2} d x$ is the usual $H_{0}^{1}$-norm, $|\cdot|_{q}$ denotes the $L^{q}(\Omega)$-norm, $q \in[1, \infty]$,

$$
S:=\inf _{f \in H_{0}^{1} \backslash\{0\}} \frac{\|f\|_{1}^{2}}{|f|_{1_{*}}^{2}}
$$

is the optimal Sobolev constant and the positive constant $C=C(\Omega)$ depends on (the diameter of) the set $\Omega$. Further

$$
|f|_{q, w}=\sup _{\substack{A \subseteq \Omega \\|A|>0}}|A|^{-1 / q^{\prime}} \int_{A}|f|
$$

denotes the standard weak $L^{q}$-norm, where $q^{\prime}$ is the conjugate of $q \in(1, \infty)$.

In what follows we prove an inequality similar to (2) for the higher order hilbertian Sobolev spaces $H_{0}^{K}(K \geq 1)$.

We endow $H_{0}^{K}(\Omega)$ with the scalar product

$$
(f, g)_{K}=\left\{\begin{array}{llc}
\int_{\Omega}\left(\Delta^{M} f\right)\left(\Delta^{M} g\right) & \text { if } & K=2 M \\
\int_{\Omega}\left(\nabla \Delta^{M} f\right)\left(\nabla \Delta^{M} g\right) & \text { if } & K=2 M+1
\end{array}\right.
$$

and we denote by $\|\cdot\|_{K}$ the corresponding norm. Consider the best constant of the Sobolev embedding inequality, namely

$$
S_{n, K}=\inf _{f \in H_{0}^{K} \backslash\{0\}} \frac{\|f\|_{K}^{2}}{|f|_{K_{*}}^{2}}
$$

see e.g. [22, 23]; of course $S$ depends on $n$ and on $K$ : however, since no confusion arises, we will simply denote it by $S$. It is well-known that $S$ is only attained when $\Omega$ is (almost) the entire space $\mathbb{R}^{n}$.

We have the following generalization of the Brezis-Lieb inequality (2) to the spaces $H_{0}^{K}$ : 
Theorem 1 Let $\Omega \subset \mathbb{R}^{n}$ be a bounded open set and let $K \geq 1$; then, there exists a constant $C=C(\Omega, K)>0$ such that

$$
\|f\|_{K}^{2} \geq S|f|_{K_{*}}^{2}+C|f|_{\frac{n}{n-2 K}, w}^{2} \quad \forall f \in H_{0}^{K}(\Omega) .
$$

This inequality is closely related to critical dimensions by means of the following principle: on bounded open sets, the Sobolev inequality for the embedding $H_{0}^{K} \subset L^{K_{*}}$ with optimal constant $S$ may be improved by adding an $L^{2}$-norm if and only if the space dimension is weakly critical. As this theorem does not require any positivity assumption on $f$, we think that it gives further support to the conjecture of Pucci and Serrin, according to which the dimensions $n=2 K+1, \ldots, 4 K-1$ are critical for (1) not only in the weak sense.

The proof of (3) for positive functions $f$ may be obtained by adjusting the proof in [5], see Section 2; the difficulty arises when we wish to prove it for any $f$ (possibly changing sign) because we can no longer replace $f$ with $|f|$. Instead, we decompose $f=f_{1}+f_{2}$ with respect to the cone of nonnegative functions $\mathcal{K}$ and its dual cone $\mathcal{K}^{\prime} \subset H_{0}^{K}$, the cone of weak subsolutions of the Dirichlet problem for the polyharmonic operator $(-\Delta)^{K}: f_{1} \in \mathcal{K}, f_{2} \in \mathcal{K}^{\prime}$. In those domains where a comparison for polyharmonic Dirichlet problems holds (e.g. in balls, which is sufficient here), one can even conclude that $f_{2}$ has a sign: $f_{2} \leq 0$, see Lemma 2 below. This allows us to prove (3) for all $f \in H_{0}^{K}(\Omega)$ in Section 3 where we also give further applications of this decomposition method, see Remark 1.

Similar phenomena appear for the quasilinear degenerate elliptic equation

$$
\left\{\begin{array}{l}
-\Delta_{p} u=\lambda|u|^{p-2} u+|u|^{p^{*}-2} u \quad \text { in } \Omega \\
u=0 \quad \text { on } \partial \Omega
\end{array}\right.
$$

where $\Delta_{p} u=\operatorname{div}\left(|\nabla u|^{p-2} \nabla u\right), n>p>1$ and $p^{*}=\frac{n p}{n-p}$. We refer to [1] for the variational characterization of the first generalized eigenvalue of $-\Delta_{p}$ and for the proof that it is simple and isolated. In this case, it is known [11] that (4) admits a positive solution for all $\lambda \in\left(0, \lambda_{1}\right)$ whenever $n \geq p^{2}$ and for any bounded smooth domain $\Omega$ : positive solutions of (4) in $B_{1}$ are necessarily radial in view of [7]; moreover, extending in an obvious way the definition of Pucci-Serrin, the critical 
dimensions are known to be $p<n<p^{2}$, see [9]. For further results with more general lower order terms see also [2].

In [10] one can find the following result: for any bounded open set $\Omega \subset \mathbb{R}^{n}$ and for all $q \in\left[1, \frac{n(p-1)}{n-p}\right)$ there exists a constant $C_{q}=C_{q}(n, q, \Omega)>0$ such that

$$
\|f\|_{W_{0}^{1, p}}^{p} \geq S|f|_{p^{*}}^{p}+C_{q}|f|_{q}^{p} \quad \forall f \in W_{0}^{1, p}(\Omega) ;
$$

moreover, $C_{q} \rightarrow 0$ as $q \rightarrow \frac{n(p-1)}{n-p}$. The optimal Sobolev constant here is defined analogously by

$$
S=S_{n, p}=\inf _{f \in W_{0}^{1, p} \backslash\{0\}} \frac{\|f\|_{W_{0}^{1, p}}^{p}}{|f|_{p^{*}}^{p}} .
$$

Then again the inequality (5) is related to the critical dimensions by means of the following principle: the remainder term in the r.h.s. of (5) contains the corresponding "linear" norm $L^{p}$ if and only if the dimension $n$ is critical.

\section{Proof of (3) for positive functions}

Let $\Omega$ be as in the statement of Theorem 1; then there exists $R>0$ such that $\Omega \subseteq$ $B_{R}$ where $B_{R}$ denotes the ball centered at the origin with radius $R$. If we extend by 0 in $B_{R} \backslash \Omega$ any function $f \in H_{0}^{K}(\Omega)$ then we see that $H_{0}^{K}(\Omega) \subseteq H_{0}^{K}\left(B_{R}\right)$ : therefore, it suffices to prove Theorem 1 in the case where $\Omega=B_{R}$ for some $R>0$.

Consider the convex closed cone of positive functions

$$
\mathcal{K}=\left\{v \in H_{0}^{K}\left(B_{R}\right) ; v(x) \geq 0 \text { for a.e. } x \in B_{R}\right\} ;
$$

we first prove inequality (3) in the case of positive functions $f$ in $B_{R}$ : this may be obtained by slight modifications of the arguments in [5].

Lemma 1 There exists a constant $\bar{C}=\bar{C}(R)>0$ such that

$$
\|f\|_{K}^{2} \geq S|f|_{K_{*}}^{2}+\bar{C}|f|_{\bar{n}}^{2}{ }_{n-2 K}, w \quad \forall f \in \mathcal{K} .
$$

Proof. Let $g \in L^{\infty}\left(B_{R}\right), g \not \equiv 0$ and $g \geq 0$ a.e., and let $u$ be a solution of the problem

$$
\left\{\begin{array}{l}
(-\Delta)^{K} u=g \quad \text { in } B_{R} \\
D^{k} u=0 \quad \text { on } \partial B_{R} \quad k=0, \ldots, K-1 ;
\end{array}\right.
$$


then, $u \in H_{0}^{K} \cap L^{\infty}\left(B_{R}\right)$. Take $f \in \mathcal{K} \backslash\{0\}$ and consider the (entire) function

$$
\phi= \begin{cases}f-u+|u|_{\infty} & \text { in } B_{R} \\ |u|_{\infty} P_{K}\left(\frac{R}{|x|}\right) & \text { in } B_{R}^{c}\end{cases}
$$

where $P_{K}$ is the polynomial of degree $n-K-1$ whose lowest power is $n-2 K$ and satisfying

$$
P_{K}(1)=1 \quad P_{K}^{(k)}(1)=0 \quad k=1, \ldots, K-1 ;
$$

for instance, if $K=2$, then $P_{2}(s)=(n-3) s^{n-4}-(n-4) s^{n-3}$.

Note that $\phi \in \mathcal{D}^{K, 2}\left(\mathbb{R}^{n}\right)$ : hence we may write the Sobolev inequality for $\phi$ on $\mathbb{R}^{n}$ and obtain (for some $C=C(n, R)>0$ )

$$
\|f-u\|_{K}^{2}+C|u|_{\infty}^{2} \geq S|f|_{K_{*}}^{2}
$$

where we used the fact that both $f \geq 0$ and $-u+|u|_{\infty} \geq 0$ a.e. in $B_{R}$. Therefore, we obtain

$$
\|f\|_{K}^{2}+\|u\|_{K}^{2}-2(f, u)_{K}+C|u|_{\infty}^{2} \geq S|f|_{K_{*}}^{2}
$$

and hence

$$
\|f\|_{K}^{2} \geq 2 \int_{B_{R}} f g-\left(\|u\|_{K}^{2}+C|u|_{\infty}^{2}\right)+S|f|_{K_{*}}^{2} ;
$$

replacing $g, u$ with $\lambda g, \lambda u$ the previous inequality remains true for all $\lambda>0$ : then, taking into account that for all $a \geq 0$ and $b>0$ we have $\max _{\lambda \geq 0}\left(a \lambda-b \lambda^{2}\right)=\frac{a^{2}}{4 b}$, we obtain

$$
\|f\|_{K}^{2} \geq S|f|_{K_{*}}^{2}+\frac{\left(\int_{B_{R}} f g\right)^{2}}{\|u\|_{K}^{2}+C|u|_{\infty}^{2}} .
$$

Let $A \subseteq B_{R},|A|>0$ and take $g=\mathbf{1}_{A}$ (the characteristic function of $A$ ) in (7); then, we obviously have

$$
\int_{B_{R}} f g=\int_{A} f .
$$

Moreover, if $\gamma=\sqrt[n]{\frac{n}{\omega_{n}}|A|}, \omega_{n}=\int_{\partial B_{1}} d \omega(x)$ and $\Gamma_{R}$ denotes the Green function corresponding to $(-\Delta)^{K}$ in $B_{R}$, then for a.e. $x \in B_{R}$ we have by Boggio's explicit formula [4, p. 126] for $\Gamma_{R}$

$$
|u(x)|=\left|\int_{B_{R}} \Gamma_{R}(x, y) \cdot \mathbf{1}_{A}(y) d y\right| \leq C(n, K) \int_{A}|x-y|^{2 K-n} d y
$$




$$
\begin{gathered}
\leq C(n, K) \int_{B_{\gamma}(x)}|x-y|^{2 K-n} d y \\
\leq C(n, K) \int_{0}^{\gamma} \rho^{n-1} \rho^{2 K-n} d \rho=C(n, K)|A|^{2 K / n} .
\end{gathered}
$$

This proves that

$$
|u|_{\infty} \leq C(n, K)|A|^{2 K / n} .
$$

Finally, by Hölder and Sobolev inequalities, we have

$$
\|u\|_{K}^{2}=(u, u)_{K}=\int_{A} u \leq|u|_{K_{*}}|A|^{(n+2 K) / 2 n} \leq C(n, K)\|u\|_{K}|A|^{(n+2 K) / 2 n} ;
$$

therefore,

$$
\|u\|_{K}^{2} \leq C(n, K)|A|^{1+2 K / n} .
$$

Since $|A| \leq\left|B_{R}\right| \leq C_{n} R^{n}$, from (9) and (10) we get

$$
\|u\|_{K}^{2}+C|u|_{\infty}^{2} \leq C(n, K, R)|A|^{4 K / n},
$$

which, together with (8), may be replaced into (7) to obtain (6) from the arbitrariness of $A$.

\section{The decomposition method}

Consider now the dual cone of $\mathcal{K}$, namely

$$
\mathcal{K}^{\prime}=\left\{w \in H_{0}^{K}\left(B_{R}\right) ;(w, v)_{K} \leq 0 \forall v \in \mathcal{K}\right\} ;
$$

in order to prove that (3) holds for any $f \in H_{0}^{K}\left(B_{R}\right)$ we first show that $\mathcal{K}^{\prime}$ is contained in the cone of negative functions:

Lemma 2 If $w \in \mathcal{K}^{\prime}$, then $w(x) \leq 0$ for a.e. $x \in B_{R}$.

Proof. Let $w \in \mathcal{K}^{\prime}$; take an arbitrary function $h \in C_{0}^{\infty}\left(B_{R}\right) \cap \mathcal{K}$ and consider the problem

$$
\left\{\begin{array}{l}
(-\Delta)^{K} v=h \quad \text { in } B_{R} \\
D^{k} v=0 \quad \text { on } \partial B_{R} \quad k=0, \ldots, K-1 ;
\end{array}\right.
$$


by the comparison principle (positivity of Green's function for the Dirichlet problem (11) in balls, cf. [4, p. 126]) we know that $v \in \mathcal{K}$ and therefore

$$
0 \geq(w, v)_{K}=\int_{B_{R}} w \cdot(-\Delta)^{K} v=\int_{B_{R}} w h .
$$

We have so proved that $\int w h \leq 0$ for all $h \in C_{0}^{\infty}\left(B_{R}\right) \cap \mathcal{K}$; by density we obtain that $\int w h \leq 0$ for all $h \in L^{2}\left(B_{R}\right)$ such that $h(x) \geq 0$ a.e. and this yields $w(x) \leq 0$ a.e.

Now take any function $f \in H_{0}^{K}\left(B_{R}\right)$; by the Proposition in [19] we infer that there exists a unique couple $\left(f_{1}, f_{2}\right) \in \mathcal{K} \times \mathcal{K}^{\prime}$ such that $f=f_{1}+f_{2}$ and $\left(f_{1}, f_{2}\right)_{K}=0$ : then

$$
\|f\|_{K}^{2}=\left\|f_{1}\right\|_{K}^{2}+\left\|f_{2}\right\|_{K}^{2}
$$

Since $f_{1}(x) \geq 0$ and $f_{2}(x) \leq 0$ (by Lemma 2) for a.e. $x \in B_{R}$ we have $\mid f_{1}(x)+$ $f_{2}(x) \mid \leq \max \left\{\left|f_{1}(x)\right|,\left|f_{2}(x)\right|\right\}$ and

$$
\left|f_{1}(x)+f_{2}(x)\right|^{r} \leq \max \left\{\left|f_{1}(x)\right|^{r},\left|f_{2}(x)\right|^{r}\right\} \leq\left|f_{1}(x)\right|^{r}+\left|f_{2}(x)\right|^{r}
$$

for all $r>0$; furthermore, if $r \geq 2$ we obtain

$$
|f|_{r}^{2}=\left(\int_{B_{R}}\left|f_{1}+f_{2}\right|^{r}\right)^{2 / r} \leq\left(\int_{B_{R}}\left|f_{1}\right|^{r}+\int_{B_{R}}\left|f_{2}\right|^{r}\right)^{2 / r} \leq\left|f_{1}\right|_{r}^{2}+\left|f_{2}\right|_{r}^{2} .
$$

By Lemma 1 we know that (6) holds for $f_{1}$ and $f_{2}$ : therefore, by (12) and (13), we get

$$
\begin{aligned}
\|f\|_{K}^{2} & =\left\|f_{1}\right\|_{K}^{2}+\left\|f_{2}\right\|_{K}^{2} \geq S\left(\left|f_{1}\right|_{K_{*}}^{2}+\left|f_{2}\right|_{K_{*}}^{2}\right)+\bar{C}\left(\left|f_{1}\right|_{\frac{n}{n-2 K}, w}^{2}+\left|f_{2}\right|_{\frac{n}{n-2 K}, w}^{2}\right) \\
& \geq S|f|_{K_{*}}^{2}+C|f|_{\frac{n}{n-2 K}, w}^{2},
\end{aligned}
$$

with $C=\bar{C} / 2$. This proves (3) for all $f \in H_{0}^{K}\left(B_{R}\right)$.

Remark 1 When dealing with weak solutions of second order elliptic equations one has to work in $H_{0}^{1}$. Here one usually decomposes $f=f^{+}+f^{-},|f|=f^{+}-f^{-}$, and $f^{+}, f^{-},|f|$ are again functions in $H_{0}^{1}$. This simple trick and its refinements (truncation on level sets) may be viewed as basis for the Stampacchia maximum principle and even for the de Giorgi-Nash-Moser theory. As mentioned above this decomposition is no longer admissible in higher order Sobolev spaces $H_{0}^{K}, K \geq 2$. 
In our opinion this is the reason that only little is known about nonlinear higher order equations compared with second order equations.

The decomposition method of Moreau together with the comparison principle of Boggio may in some situations in $H_{0}^{K}(K \geq 2)$ substitute the decomposition in positive and negative part just mentioned. A first application to a fourth order eigenvalue problem can e.g. be found in [17].

Here we want to present another example. In the previous paper [14], Grunau constructs a positive solution to the semilinear Dirichlet problem (1) in balls $B$, but he leaves open the question whether this solution may be found (up to scaling) as minimizer of the variational problem

$$
S_{\lambda}=\min _{v \in H_{0}^{K}(B) \backslash\{0\}} \frac{\|v\|_{K}^{2}-\lambda|v|_{2}^{2}}{|v|_{K_{*}}^{2}}
$$

where $\lambda \in\left(0, \lambda_{1}\right)$. Now it is easy to show that, at least in a ball, any minimizer (for existence see also [14]) of this problem must be positive. Indeed, assume that $u$ is a minimizer and $u \nsupseteq 0$ : then we decompose as above $u=u_{1}+u_{2}, u_{1} \in \mathcal{K}, u_{2} \in \mathcal{K}^{\prime}$. Again by Lemma 2 we conclude $u_{2} \leq 0, u_{2} \not \equiv 0$. So replacing $u$ with the positive function $u_{1}-u_{2}$ would strictly increase the $L^{2}$-norm in the numerator and the $L^{K_{*}}$ norm in the denominator. By orthogonality we have $\left\|u_{1}+u_{2}\right\|_{K}^{2}=\left\|u_{1}-u_{2}\right\|_{K}^{2}$. As $\lambda>0$ the fraction would strictly decrease, a contradiction!

An even simpler reasoning gives besides the positivity of the first eigenfunction also the simplicity of the first Dirichlet eigenvalue of $(-\Delta)^{K}$ in balls without referring to Krein-Rutman's or Jentzsch's theorem.

Acknowledgement. The authors are grateful to Patrizia Pucci for her useful suggestions on a preliminary version of the manuscript.

\section{References}

[1] A. Anane, Simplicité et isolation de la première valeur propre du p-laplacien avec poids, C. R. Acad. Sci. Paris 305, 1987, 725-728

[2] G. Arioli, F. Gazzola, Some results on p-Laplace equations with a critical growth term, Diff. Int. Eq. 11, 1998, 311-326

[3] F. Bernis, H.-Ch. Grunau, Critical exponents and multiple critical dimensions for polyharmonic operators, J. Diff. Eq. 117, 1995, 469-486 
[4] T. Boggio, Sulle funzioni di Green d'ordine m, Rend. Circ. Mat. Palermo 20, 1905, 97-135

[5] H. Brezis, E. Lieb, Sobolev inequalities with remainder terms, J. Funct. Anal. 62, $1985,73-86$

[6] H. Brezis, L. Nirenberg, Positive solutions of nonlinear elliptic equations involving critical Sobolev exponents, Comm. Pure Appl. Math. 36, 1983, 437-477

[7] F. Brock, Radial symmetry for nonnegative solutions of semilinear elliptic equations involving the p-Laplacian, Progress in Partial Differential Equations, Vol.1 (Pont-àMousson 1997) Pitman Res. Notes Math. Ser. 383, 1998, 46-57

[8] D.E. Edmunds, D. Fortunato, E. Jannelli, Critical exponents, critical dimensions and the biharmonic operator, Arch. Rat. Mech. Anal. 112, 1990, 269-289

[9] H. Egnell, Elliptic boundary value problems with singular coefficients and critical nonlinearities, Indiana Univ. Math. J. 38, 1989, 235-251

[10] H. Egnell, F. Pacella, M. Tricarico, Some remarks on Sobolev inequalities, Nonlin. Anal. TMA 13, 1989, 671-681

[11] J.P. García Azorero, I. Peral Alonso, Existence and nonuniqueness for the pLaplacian: nonlinear eigenvalues, Comm. Part. Diff. Eq. 12, 1987, 1389-1430

[12] F. Gazzola, Critical growth problems for polyharmonic operators, Proc. Roy. Soc. Edinburgh 128A, 1998, 251-263

[13] H.-Ch. Grunau, Critical exponents and multiple critical dimensions for polyharmonic operators, Boll. Un. Mat. Ital. 7, 9-B, 1995, 815-847

[14] H.-Ch. Grunau, Positive solutions to semilinear polyharmonic Dirichlet problems involving critical Sobolev exponents, Calc. Var. 3, 1995, 243-252

[15] H.-Ch. Grunau, On a conjecture of P. Pucci and J. Serrin, Analysis, 16, 1996, 399-403

[16] E. Jannelli, The role played by space dimension in elliptic critical problems, to appear in J. Diff. Eq.

[17] E. Miersemann, Über positive Lösungen von Eigenwertgleichungen mit Anwendungen auf elliptische Gleichungen zweiter Ordnung und auf ein Beulproblem für die Platte, Z. Angew. Math. Mech. ZAMM 59, 1979, 189-194

[18] E. Mitidieri, On the definition of critical dimension, unpublished manuscript, 1993

[19] J.J. Moreau, Décomposition orthogonale d'un espace hilbertien selon deux cônes mutuellement polaires, C. R. Acad. Sci. Paris 255, 1962, 238-240

[20] P. Pucci, J. Serrin, A general variational identity, Indiana Univ. Math. J. 35, 1986, 681-703 
[21] P. Pucci, J. Serrin, Critical exponents and critical dimensions for polyharmonic operators, J. Math. Pures Appl. 69, 1990, 55-83

[22] C.A. Swanson, The best Sobolev constant, Appl. Anal. 47, 1992, 227-239

[23] G. Talenti, Best constant in Sobolev inequality, Ann. Mat. Pura Appl. 110, 1976, 353-372 